BNL-96825-2012-IR

$\mathrm{C}-\mathrm{A} / \mathrm{AP} / \# 446$

Jan. 2012

\title{
Upgrade scenario for the RHIC collimation system
}

\section{G. Robert-Demolaize, A. Drees}

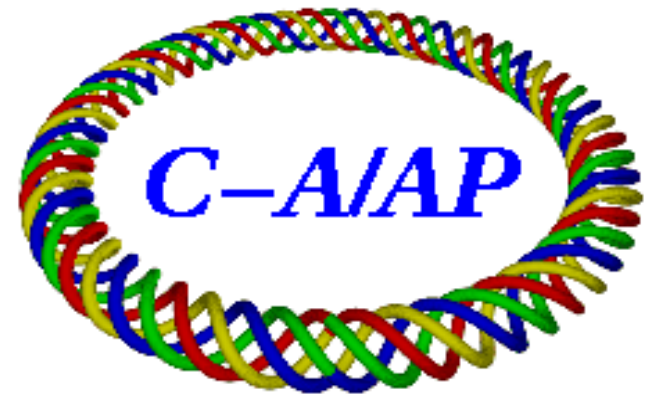

Collider-Accelerator Department Brookhaven National Laboratory Upton, NY 11973

Notice: This document has been authorized by employees of Brookhaven Science Associates, LLC under Contract No. DE-AC02-98CH10886 with the U.S. Department of En ergy. The United States Government retains a nonexclusive, paid-up, irrevocable, world-wide license to publish or reproduce the published form of this document, or allow others to do so, for United States Government purposes. 


\title{
UPGRADE SCENARIO FOR THE RHIC COLLIMATION SYSTEM
}

\author{
G. Robert-Demolaize, A. Drees
}

\section{Abstract}

The RHIC collimation system is used to reduce background levels in both STAR and PHENIX detectors. With a push for higher luminosity in the near future, it becomes critical to check if and how the level of performance of the collimators can be improved. The following reviews a proposal for additional collimators placed further downstream of the current system and designed to intercept the tertiary halo coming out of the IR8 insertion before it can reach the triplet quadrupoles in either STAR or PHENIX.

\section{INTRODUCTION}

Using live data from previous runs, the RHIC collimation system allowed benchmarking the extended version of SixTrack for collimation studies [1]. This software can now be used to study an upgrade scenario to the current system. Table 1 lists the center position and active plane (i.e. the beam direction that is intercepted by the collimator jaw) for each collimator in both Blue and Yellow beamlines in the main ring of RHIC. The collimation system is located between Q3 and Q4 downstream of the PHENIX experiment in IR8, with all four collimators installed over a drift space of roughly 20 meters.

Table 1: Location (from IP6) and active plane of RHIC collimators in the current system.

\begin{tabular}{|c|c|c|c|c|}
\cline { 2 - 5 } \multicolumn{1}{c|}{} & \multicolumn{2}{c|}{ Blue } & \multicolumn{2}{c|}{ Yellow } \\
\hline Name & $\mathrm{S}[\mathrm{m}]$ & Plane & $\mathrm{S}[\mathrm{m}]$ & Plane \\
\hline COL0 & 680.752 & Hor. + Vert. & 3236.649 & Hor. + Vert. \\
\hline COLH1 & 690.533 & Horizontal & 3246.430 & Horizontal \\
\hline COLV1 & 696.706 & Vertical & 3252.603 & Vertical \\
\hline COLH2 & 697.728 & Horizontal & 3253.625 & Horizontal \\
\hline
\end{tabular}

Table 2: Twiss function $\beta_{x, y}$ for Run FY09 at $\mathrm{E}_{\text {store }}=100 \mathrm{GeV}$ at the RHIC collimators.

\begin{tabular}{|c|c|c|c|c|}
\cline { 2 - 5 } \multicolumn{1}{c|}{} & \multicolumn{2}{c|}{ Blue } & \multicolumn{2}{c|}{ Yellow } \\
\hline Name & $\beta_{x}[\mathrm{~m}]$ & $\beta_{y}[\mathrm{~m}]$ & $\beta_{x}[\mathrm{~m}]$ & $\beta_{y}[\mathrm{~m}]$ \\
\hline COL0 & 1486.007 & 483.661 & 1534.254 & 504.750 \\
\hline COLH1 & 845.084 & 201.805 & 871.956 & 210.945 \\
\hline COLV1 & 533.168 & 86.653 & 549.741 & 90.768 \\
\hline COLH2 & 488.422 & 72.266 & 503.527 & 75.737 \\
\hline
\end{tabular}

Another characteristic of the collimation for RHIC is the behavior of the optic functions along the system: as shown in Table $2, \beta_{x, y}$ decrease rapidly between the primary collimator COLO and the secondary jaws. Assuming a nor-
Table 3: Collimator openings for Run FY09 at $\mathrm{E}_{\text {store }}=100 \mathrm{GeV}$ in unit of sigmas and in millimeters. Blue and Yellow use the same normalized settings.

\begin{tabular}{|c|c|c|c|}
\hline Name & $\mathbf{N}_{x, y}\left[\sigma_{x, y}\right]$ & $\mathbf{n}_{x, y}^{\text {Blue }}[\mathbf{m m}]$ & $\mathbf{n}_{x, y}^{\text {Yellow }}[\mathbf{m m}]$ \\
\hline COL0 Hor. & 5.866 & 39.97 & 40.61 \\
\hline COL0 Vert. & 4.583 & 17.82 & 18.20 \\
\hline COLH1 & 7.533 & 38.71 & 39.32 \\
\hline COLV1 & 6.0265 & 9.92 & 10.15 \\
\hline COLH2 & 8.131 & 31.76 & 32.25 \\
\hline
\end{tabular}

malized emittance of $20 \pi$, one gets $\sigma_{x}=6.81 \mathrm{~mm}$ and $\sigma_{y}$ $=3.90 \mathrm{~mm}$ at COL0, while $\sigma_{x}=3.89 \mathrm{~mm}$ at COLH2 and $\sigma_{y}=1.65 \mathrm{~mm}$ at COLV1. Optimizing the positions of the secondary collimators to capture the halo coming from the primary can therefore prove difficult to achieve.

In order to increase the efficiency of the current system, one could find the optimal locations for additional collimators that would be designed to absorb most of the secondary halo (from COLH1, COLH2 and COLV1 for each beamline) before it reaches the triplet quadrupole in STAR or PHENIX. Such locations can be determined based on the theory of beam collimation (reviewed in [2]) and the available space in RHIC. One major change would be the use of two-sided tungsten jaws, as opposed to the current one-sided copper collimators. This would allow the system to be less sensitive to a new working point in the future.

\section{PROPOSED NEW LOCATIONS}

As mentioned above, the plan for the mask collimators is to provide additional protection against background in STAR and PHENIX. The motivation behind installing mask collimators comes from the fact that the STAR experiment is the very next insertion downstream of the collimators following the Yellow beam. The baseline for finding the optimal locations for those masks should therefore be the Yellow lattice; an equivalent solution will then be applied to the Blue lattice. The constraints to be considered are the optic functions between IR8 (collimation insertion) and IR6 (STAR insertion) and the available space for the new equipment that would have to be installed.

\section{Phase advance from $\mathrm{COLO}$}

To find the location of the new collimators (that could be called either tertiary jaws or masks), one could define a golden situation for the collimation. Let us consider the following case: the RHIC collimators are setup according 
Table 4: Mask collimator properties; the optics functions values are taken from Run FY09 at $\mathrm{E}_{\text {store }}=100 \mathrm{GeV}$ settings. S locations are in reference to IP6 going clockwise.

\begin{tabular}{|c|c|c|c|c|c|c|c|c|}
\hline Ring & Name & $\mathbf{S}[\mathbf{m}]$ & $\mathbf{L}[\mathbf{m}]$ & $\beta_{x}[\mathbf{m}]$ & $\beta_{y}[\mathbf{m}]$ & $\mathbf{N}_{x, y}$ & $\mathbf{n}_{x, y}[\mathbf{m m}]$ & Material \\
\hline \multirow{2}{*}{ Blue } & TCTH.TS1.B1 & 1129.061 & 0.45 & 25.853 & 21.625 & $\mathrm{~N}_{x}=9.658$ & $\mathrm{n}_{x}=8.68$ & Tungsten \\
\cline { 2 - 9 } & TCTV.TS1.B1 & 1132.561 & 0.45 & 37.696 & 13.703 & $\mathrm{~N}_{y}=4.655$ & $\mathrm{n}_{y}=3.05$ & Tungsten \\
\hline \multirow{2}{*}{ Yellow } & TCTH.TS2.B1 & 3684.958 & 0.45 & 24.722 & 21.891 & $\mathrm{~N}_{x}=9.767$ & $\mathrm{n}_{x}=8.58$ & Tungsten \\
\cline { 2 - 9 } & TCTV.TS2.B1 & 3688.458 & 0.45 & 35.920 & 14.130 & $\mathrm{~N}_{y}=4.650$ & $\mathrm{n}_{y}=3.09$ & Tungsten \\
\hline
\end{tabular}

to their settings during Run FY09 (see Table 3), and the beam hits the primary jaw COLO with an impact parameter of $1 \mu \mathrm{m}$ in each plane (since COL0 is L-shaped to work in both planes at once). From [2] and SixTrack sample runs, one can calculate the resulting transverse kicks $\Delta x p$ and $\Delta y p$ that a particle gets from the iteraction with the primary copper jaw:

$$
\Delta x p=\Delta y p=-155 \mu \mathrm{rad},
$$

with $x p$ and $y p$ the horizontal and vertical angle (respectively) of a particle motion along the $s$ axis. To calculate the phase advance $\Delta \mu$ generated by such a transverse kick, one can use the following normalized coordinates expression:

$$
\tan \left(\Delta \mu_{z}\right)=\frac{\Delta Z P}{Z_{\text {coll }}},
$$

with $Z(Z P)$ either of the transverse normalized coordinates $X(X P)$ or $Y(Y P)$, and $Z_{\text {coll }}$ the opening of the primary collimator in normalized coordinates in the corresponding plane. With $\beta_{z}$ and $\alpha_{z}$ the twiss functions at the location of the primary collimator and $\epsilon_{z}$ the beam emittance in the considered transverse plan, one finds:

$$
Z_{\mathrm{coll}}=\frac{z_{\mathrm{coll}}}{\sqrt{\beta_{z} \epsilon_{z}}}
$$

and:

$$
\Delta Z P=\frac{\left|\Delta \mu_{z}+\frac{\alpha_{z} z_{\text {coll }}}{\beta_{z}}\right|}{\sqrt{\epsilon_{z} / \beta_{z}}} .
$$

Inserting Eq. 3 and 4 in Eq. 2, it yelds:

$$
\tan \left(\Delta \mu_{z}\right)=\frac{\beta_{z}}{z_{\text {coll }}} .\left|\Delta \mu_{z}+\frac{\alpha_{z} z_{\text {coll }}}{\beta_{z}}\right| .
$$

With Eq. 1 and Table 2, along with the corresponding values of $\alpha_{x}$ and $\alpha_{y}$ at the location of COL0, one gets:

$$
\begin{gathered}
\Delta \mu_{x}(\text { Blue })=0.2450 \\
\Delta \mu_{y}(\text { Blue })=0.2381 \\
\Delta \mu_{x}(\text { Yellow })=0.2451 \quad \Delta \mu_{y}(\text { Yellow })=0.2386 .
\end{gathered}
$$

\section{Mechanical design and available space}

The new collimators for the considered upgrade scenario would feature two jaws per plane, centered around the beam path. The current choice for the jaw material is tungsten, which would provide very good absorption rate for the incoming secondary halo. The motivation behind tungsten is its succesful use for the LHC tertiary collimators, designed to protect the experimental triplet magnets from beam induced quenches; using tungsten to reduce halo backgrounds in RHIC experiments should therefore carry similar efficiency. The mechanical design of the collimator tank should follow the current scheme with 45 centimeters long jaws; the total flange-to-flange lenght of a collimator tank is approximately 1.5 meters.

As stated above, the critical item for this upgrade is reducing the background in STAR following the Yellow beamline, since it is the experiment immediately downstream of the current collimation system for that beam. The goal when trying to locate the new mask collimators is to find a stretch of open space large enough to fit both a horizontal and a vertical mask (plus all required space for installation purposes) upstream from the first triplet quadrupole when getting into IR6, which lattice name is $y i 6-q f 3)$. One can find two potential locations:

- between yi6 - dh9 and yi6 - qf9: roughly 7.5 meters available and $\Delta \mu_{x}(\mathrm{COL} 0)=3.1577, \Delta \mu_{y}(\mathrm{COL} 0)=$ 3.9552;

- between yi6-qd8 and yi6-qf7: roughly 13.1 meters available and $\Delta \mu_{x}(\mathrm{COL} 0)=3.4156, \Delta \mu_{y}(\mathrm{COL} 0)=$ 4.1773;

the phase advance values being calculated at the centre of the empty space. The two locations listed above are the ones that fit Eq. 6 requirement the closest. Even though the first location is the shortest, this is the location that was used for the study reported in this article, based on the fact that there is a smaller difference in phase advance compared to the target value in the horizontal plane.

Eq. 6 shows very similar values between the Blue and the Yellow beam; for practical reasons it was decided to use the location in Blue equivalent to the one selected for Yellow, which corresponds to the space between bi9-dh9 and bi9-qf9. Table 4 summarizes all the characteristics (optics and mechanical) of the mask collimators for the studied upgrade; $\mathrm{N}_{x}$ and $\mathrm{N}_{y}$ are the calculated openings of 
each mask collimator in units of sigmas using the baseline of Table 3 for the COL0 settings.

\section{SIMULATION RESULTS}

Figure 1 shows the result of simulations performed with SixTrack using Run FY09 settings and optics, respectively without and with the new mask collimator from the studied upgrade scenario, following the Yellow beam moving from left to right (i.e. going counter-clockwise around RHIC). For these simulations, we track separately the horizontal and the vertical plane of the beam, using "pencil" distributions of 24000 protons. The tracked particles are all generated as a point-like beam arriving on the primary collimator jaw with an impact parameter of $1 \mu \mathrm{m}$, meaning that all particles are generated at a normalized amplitude of (primary collimator opening) $+1 \mu \mathrm{m}$. This is done so as to simulate the interaction of the beam halo with the collimation system.

In each figure, the bars labeled "cold losses" correspond to the amount of protons lost in the machine aperture; the "collimator losses" lines list the number of protons absorbed at the collimtators, i.e. the ones suffering inelastic scattering interactions with the jaw material (copper for the existing system, tungsten for the new masks). One can clearly see the effect of the additional collimators, the losses incoming into IR6 being nearly completely removed. Table 6 provides a list of the largest losses around the ring, without and with the upgrade. Losses immediately downstream of the collimators in IR8 are identical between the 2 simulation scenarios because (1) the random seed used was the same (for practical purposes, to provide a "what would have happened" representation) and (2) losses in that area are unavoidable since they come from direct showering from the collimators to the nearby magnets located a few meters down the beamline. Predicted beam loss statistics downstream of the IR8 collimators are therefore not included for comparison in Table 6. Figure 2 shows the data of simulations for the Blue beam again with Run FY09 optics with and without the new mask collimators. Table 7 reviews the statistics for these simulations.

Table 5: Working point settings for all three simulation cases studied.

\begin{tabular}{|c|c|c|}
\cline { 2 - 3 } \multicolumn{1}{c|}{} & $\mathbf{Q}_{x}$ & $\mathbf{Q}_{y}$ \\
\hline Case 1: design working point & 28.695 & 29.685 \\
\hline Case 2: $\Delta \mathrm{Q}=+1$ & 29.695 & 30.685 \\
\hline Case 3: $\Delta \mathrm{Q}=-1$ & 27.695 & 28.685 \\
\hline
\end{tabular}

The previous results are for an ideal case only, based on optics from RHIC Run FY09. In order to assess the efficiency of the proposed upgrade, it is worth checking how the longitudinal loss pattern changes when the working point is changed. Table 5 lists the tune parameters for the ideal case and the two considered tune shifts. All other optics parameters $\left(\beta^{*}\right.$, design orbit, collimator positions) are identical. Figures 3 and 4 present the results of simulations for Case 2, while Case 3 is shown in Figures 5 and 6. All statistics are compiled in Table 8, 9, 10 and 11.

\section{CONCLUSION}

Simulations have been peformed to quantify the efficiency of additional collimator jaws in RHIC. Each figure presented in this article clearly shows that the additional mask collimators provide the expected reduction in losses around the machine, and especially to the incoming triplet to the STAR experiment (IP6), for the Yellow bema as much as for the Blue beam. Looking at compiled statistics for all three working point cases studied, proton losses around the machine are reduced by roughly one order of magnitude: at most a factor 30 for magnet losses, and at most a factor 40 for losses in spaces between magnets.

\section{REFERENCES}

[1] G. Robert-Demolaize, A. Drees, "Benchmarking of collimation tracking using RHIC beam loss data", Proc. EPAC 2008.

[2] G. Robert-Demolaize: "Design and performance optimization of the LHC collimation system", CERN-LHCPROJECT-REPORT-981, 2006. 

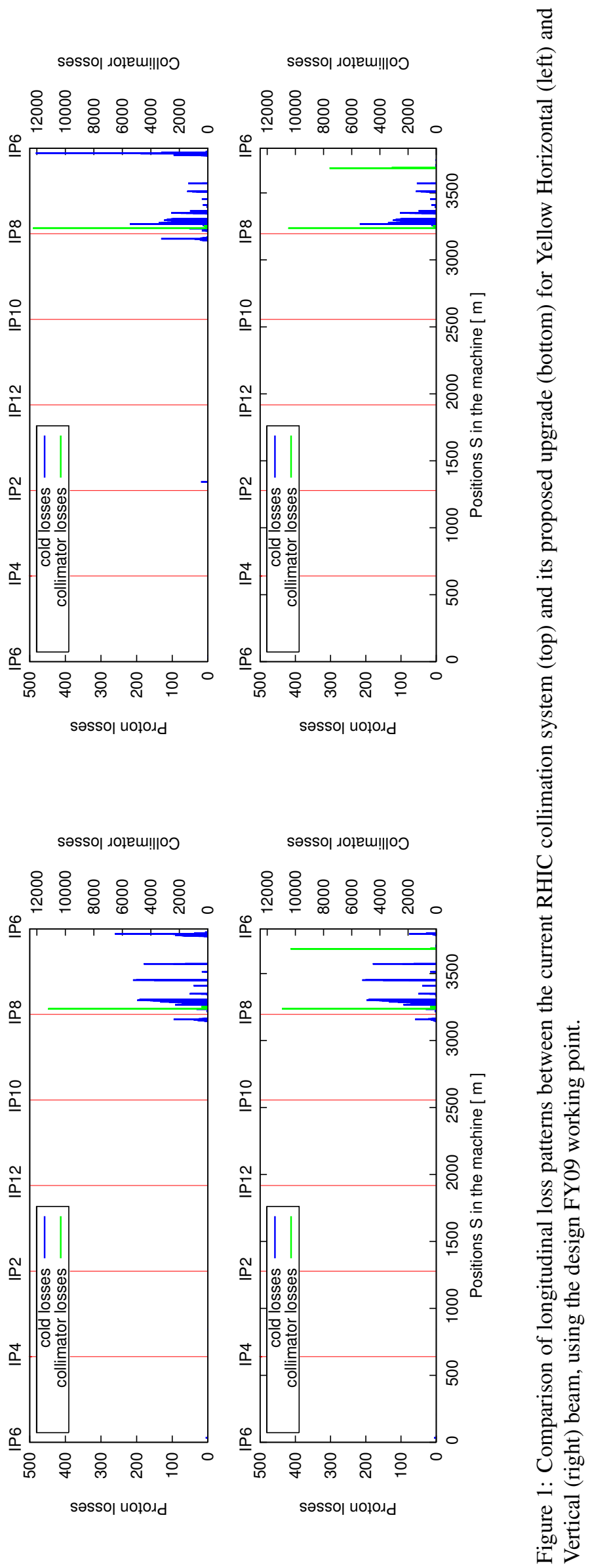

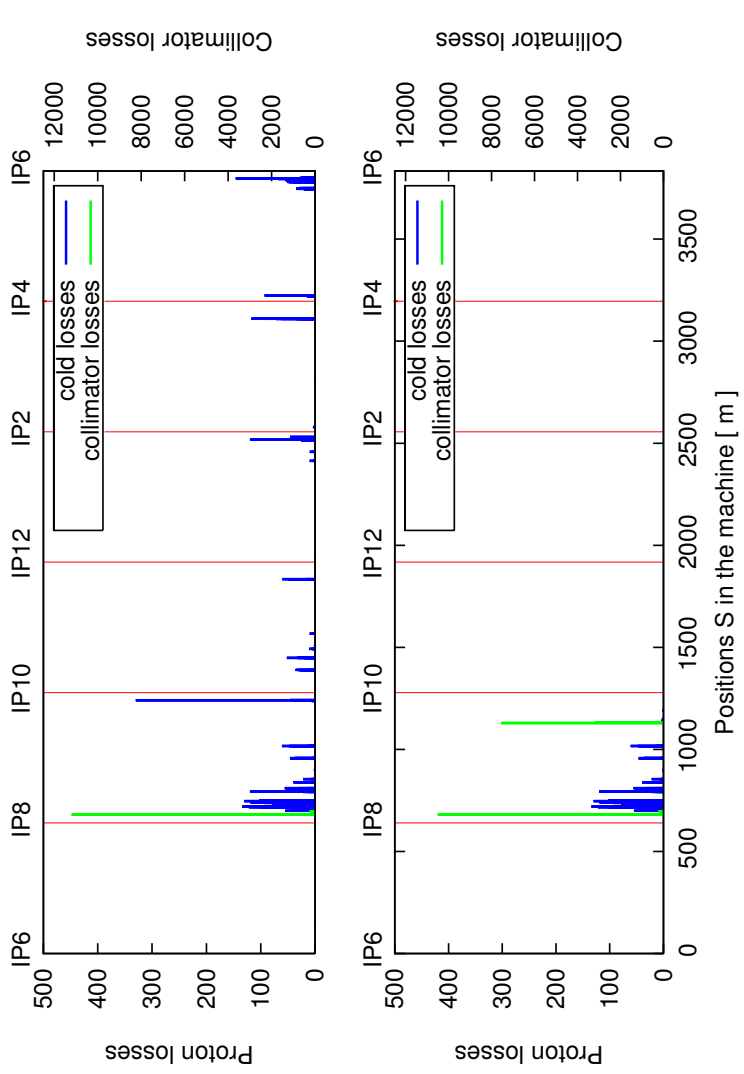

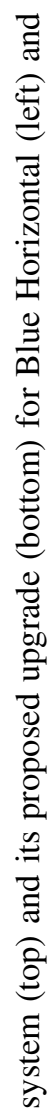
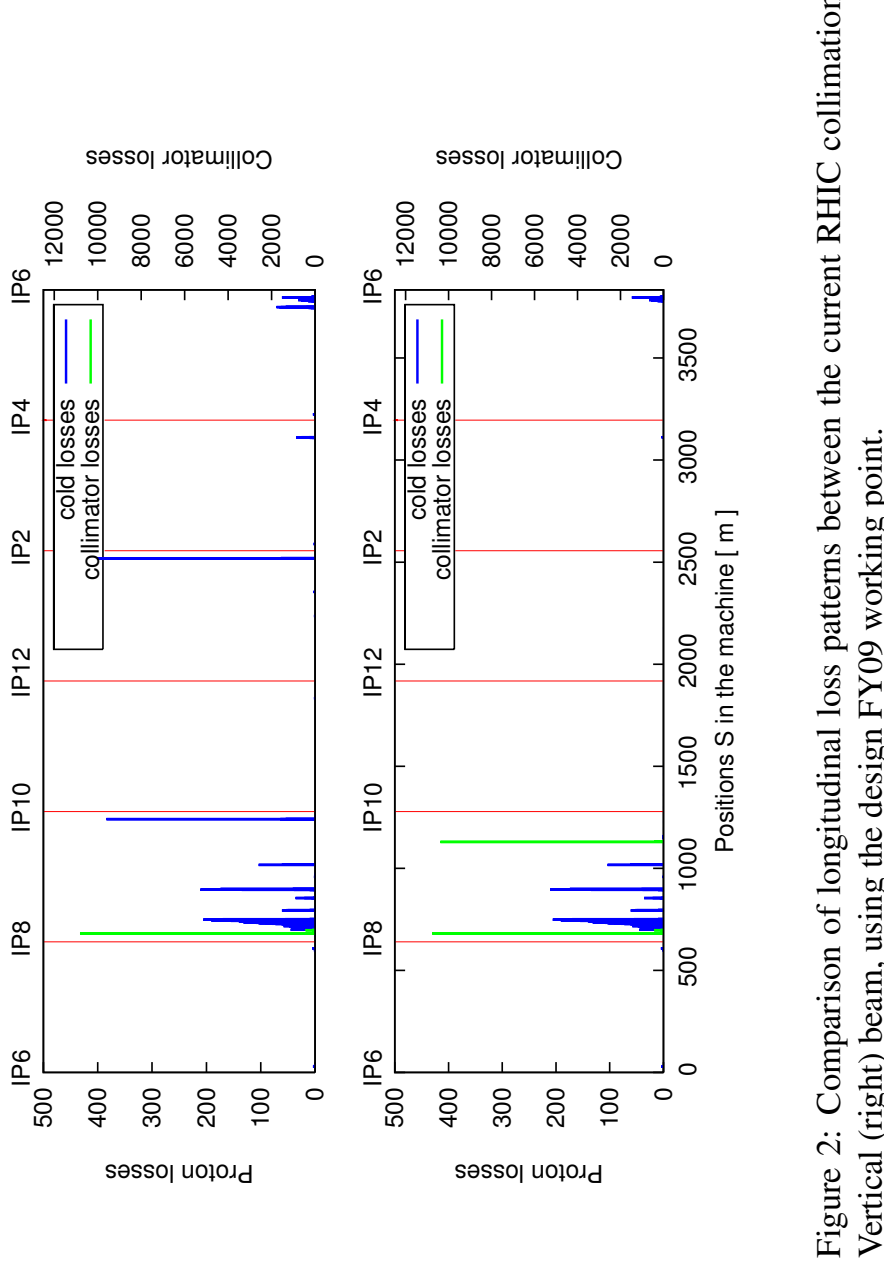


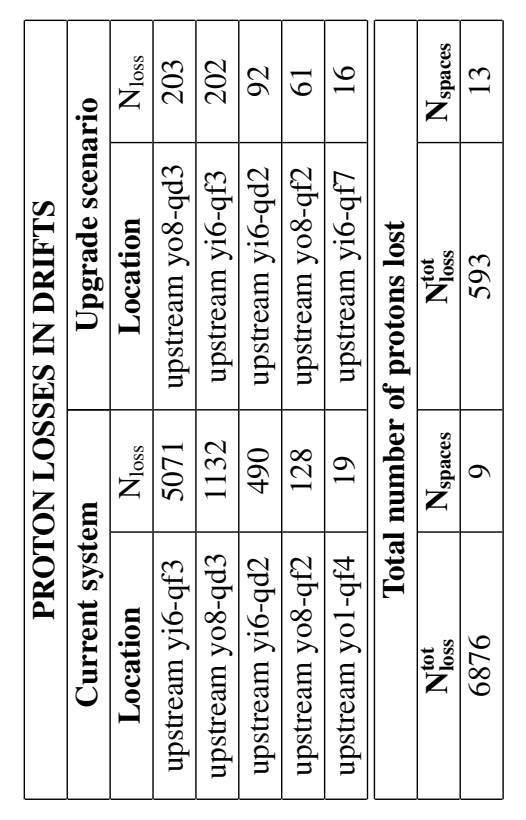

苞

悉

莺

离

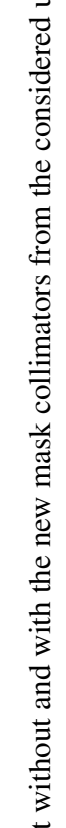

$\stackrel{\circ}{\circ}$

홍

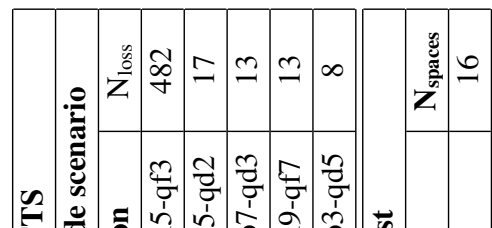

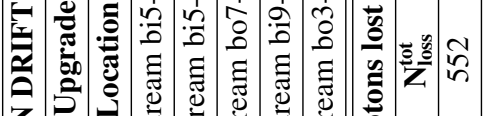

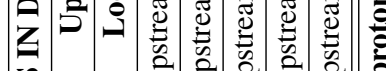

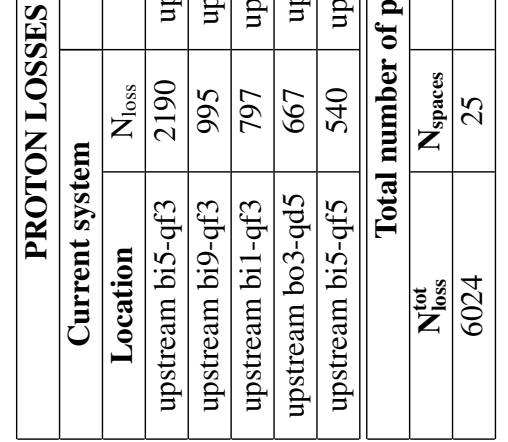

을.

흘

흘

त्र

负.

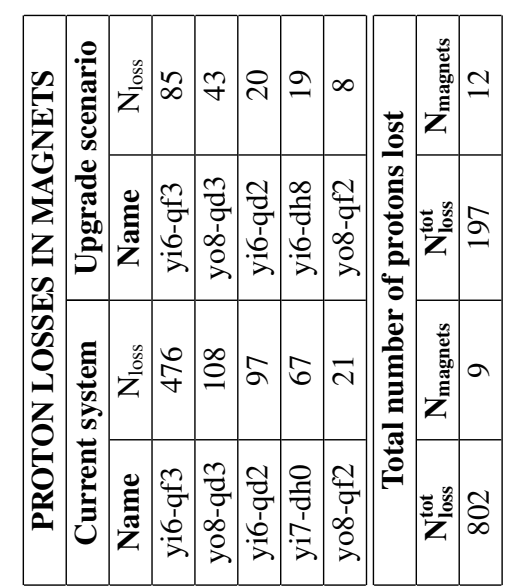

:

莙

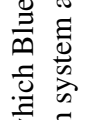

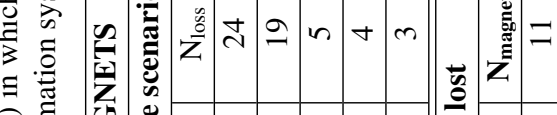

过

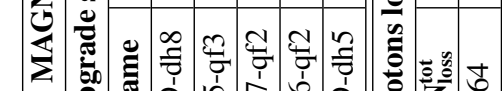

क्षे

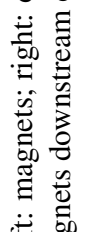

芦芯

כ

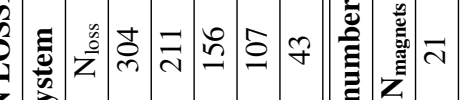

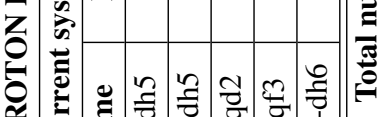

先

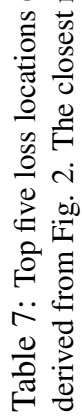



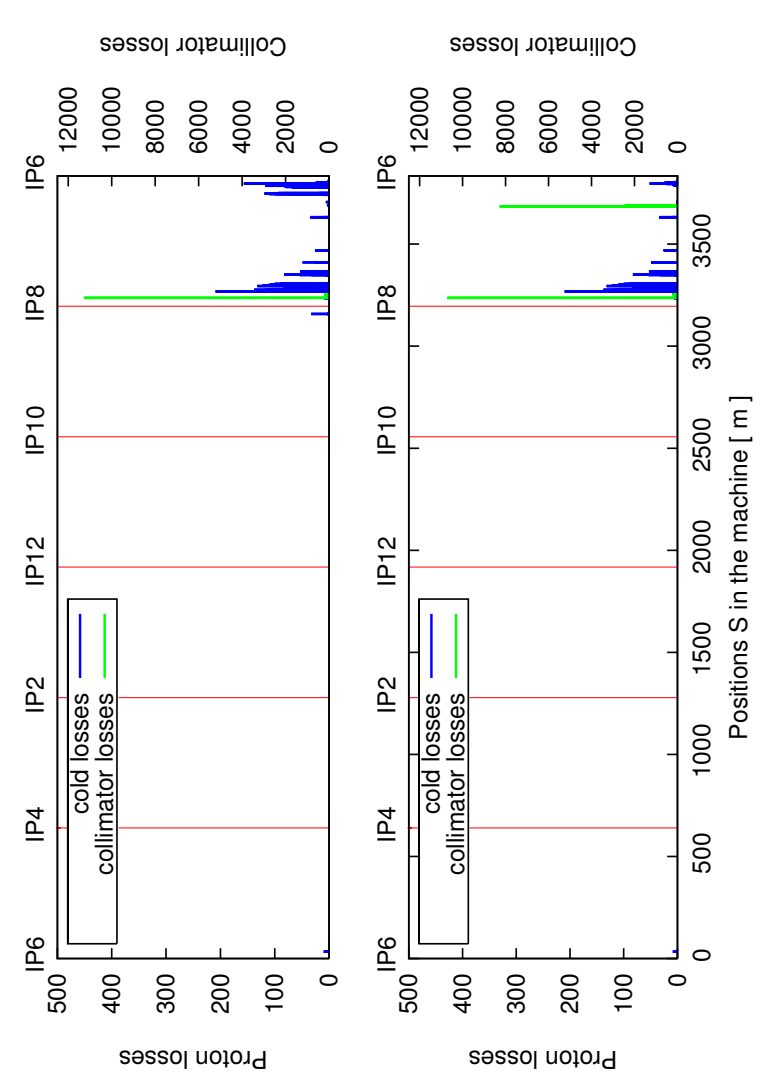

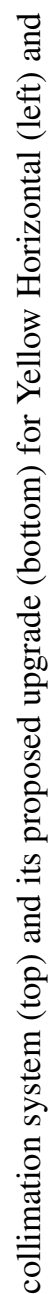
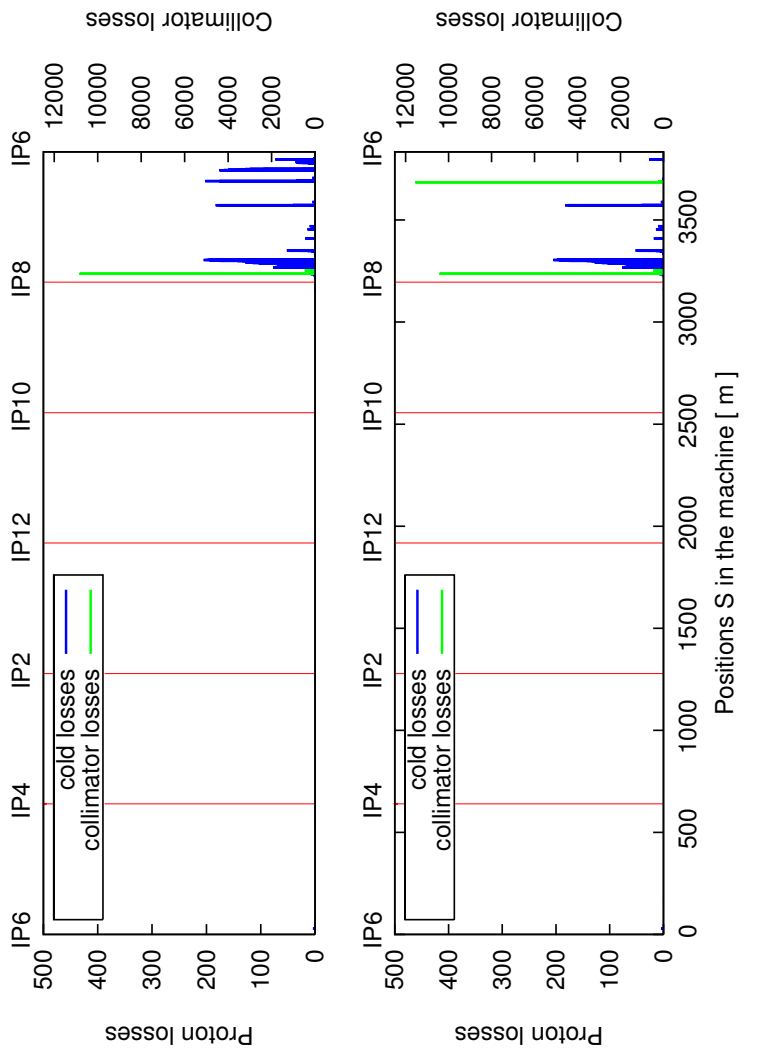

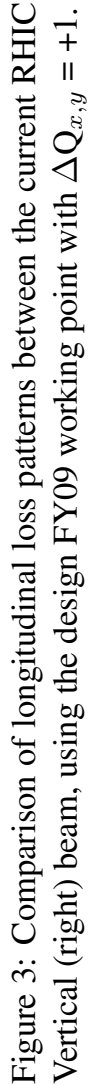



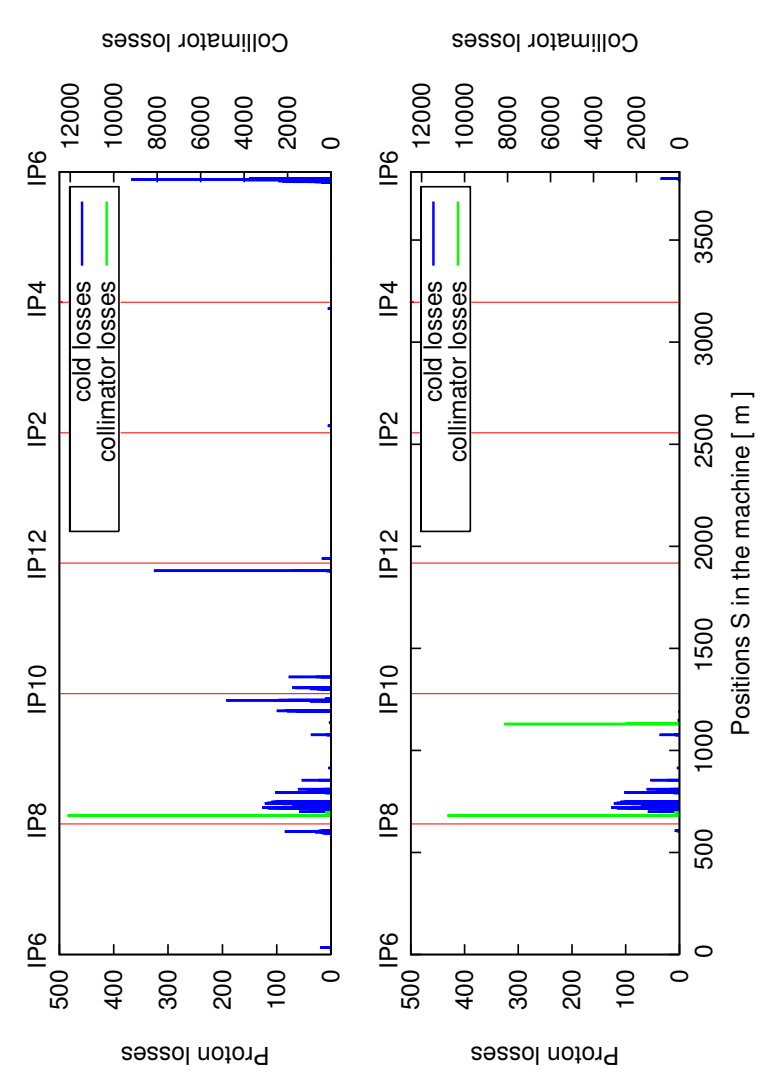

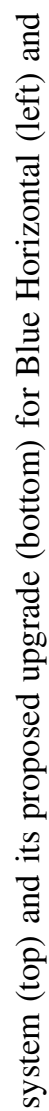
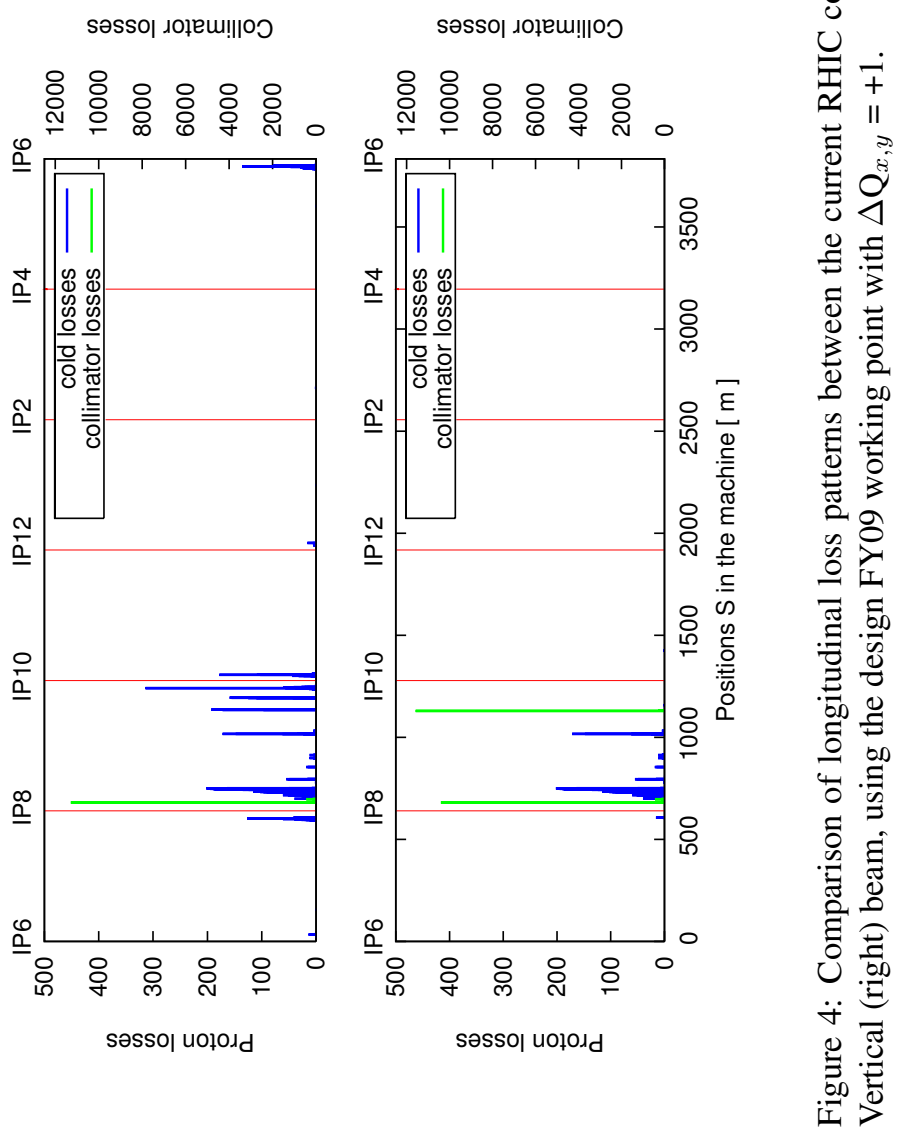


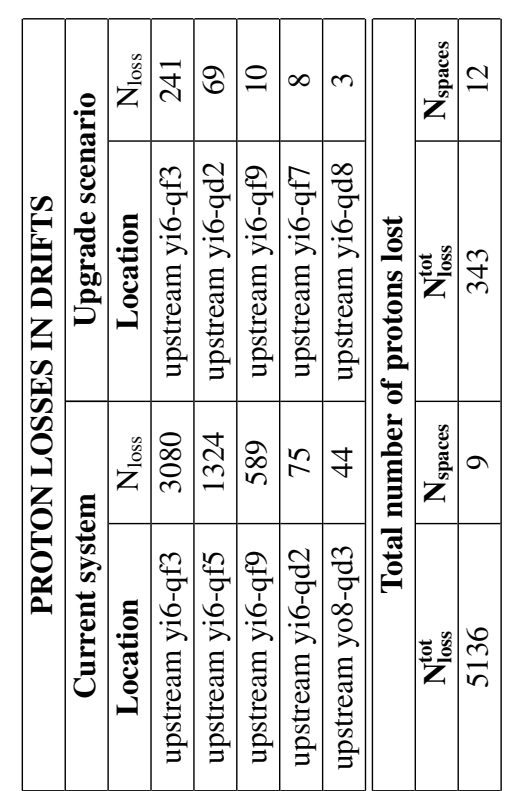

氖

苟

范

递

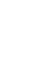

วิ

늘

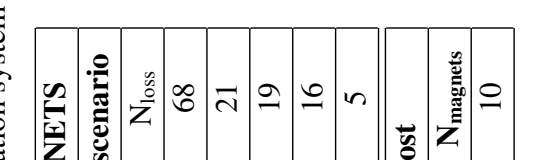



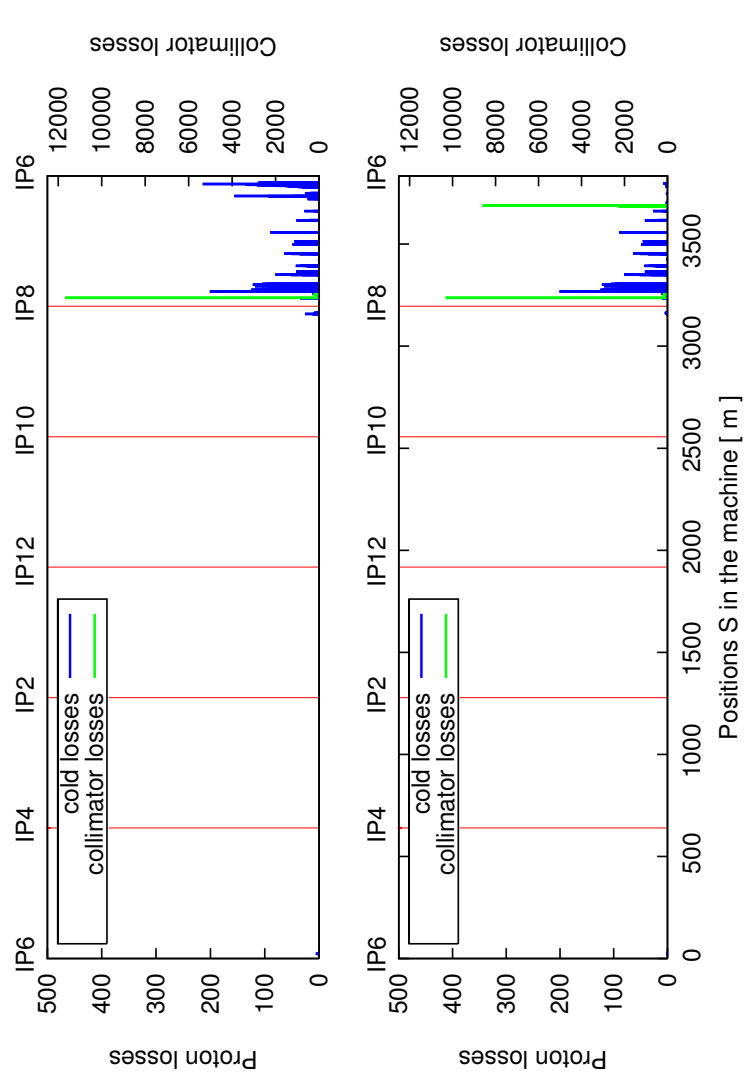

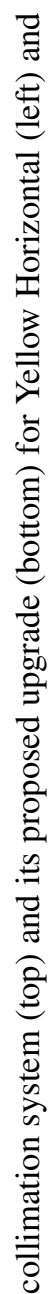
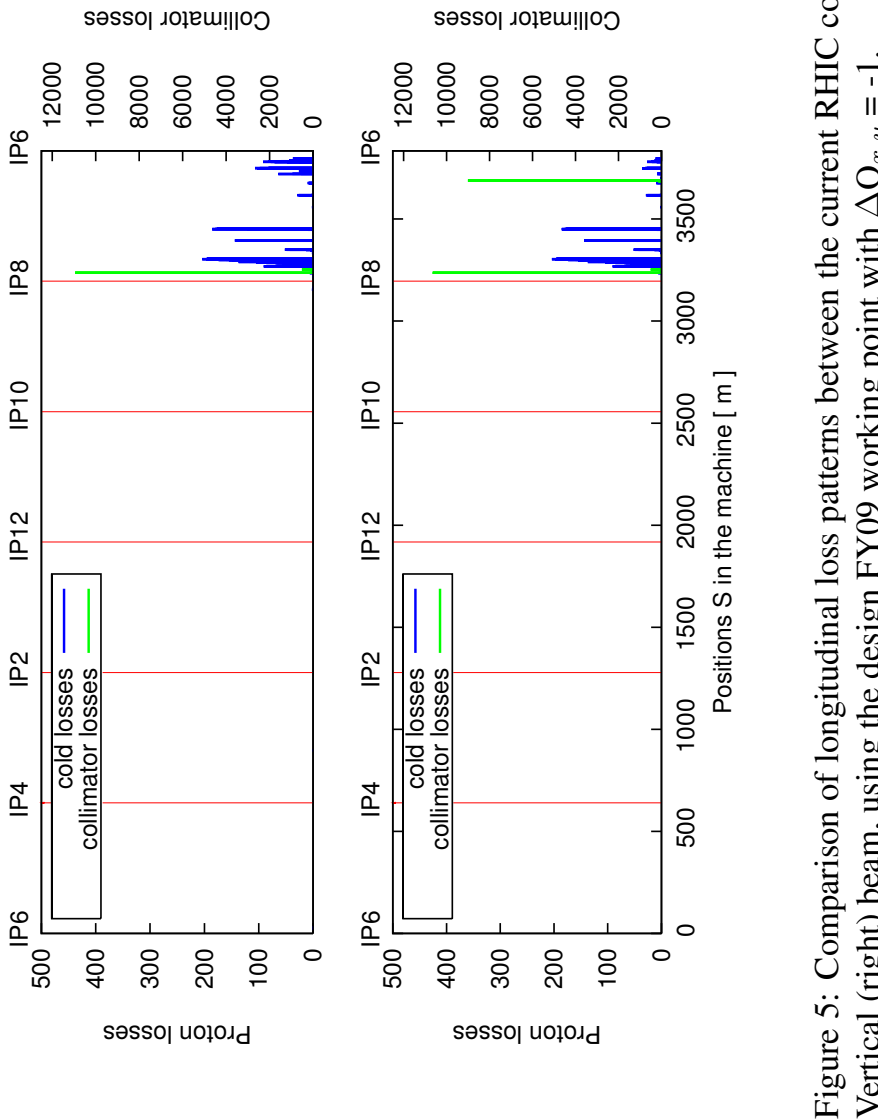

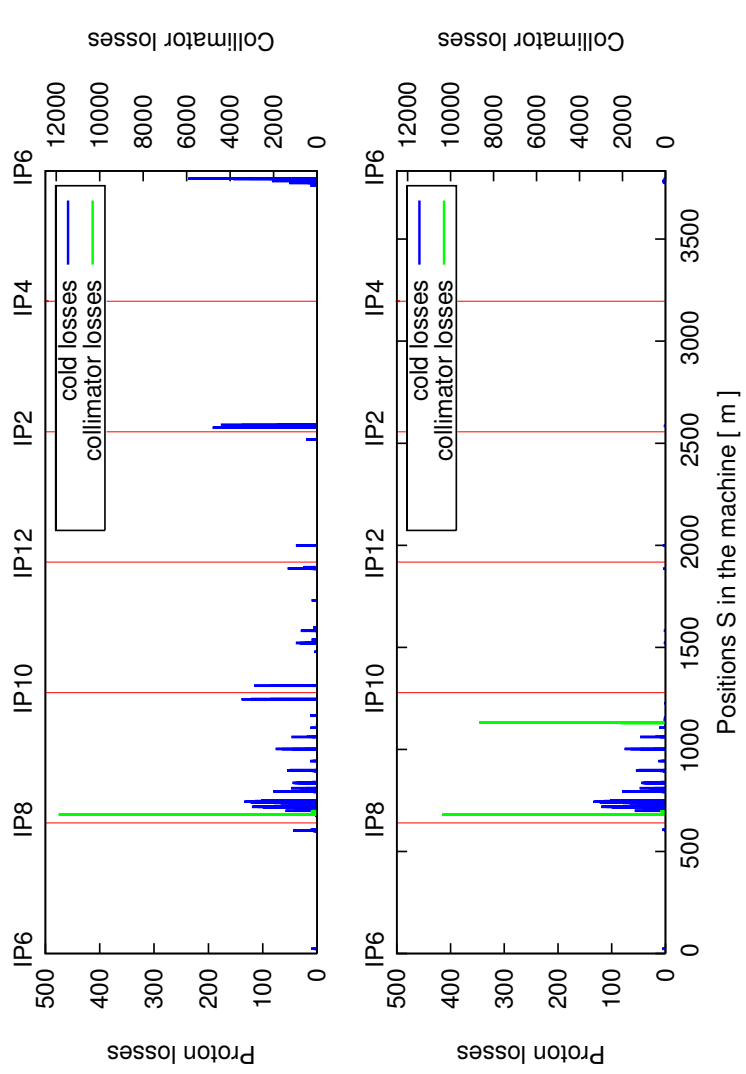

0
0
0
0
0
0
0
0
0
0
0
0
0
0
0
0
0
0
0
0
0
0
0
0
0
0
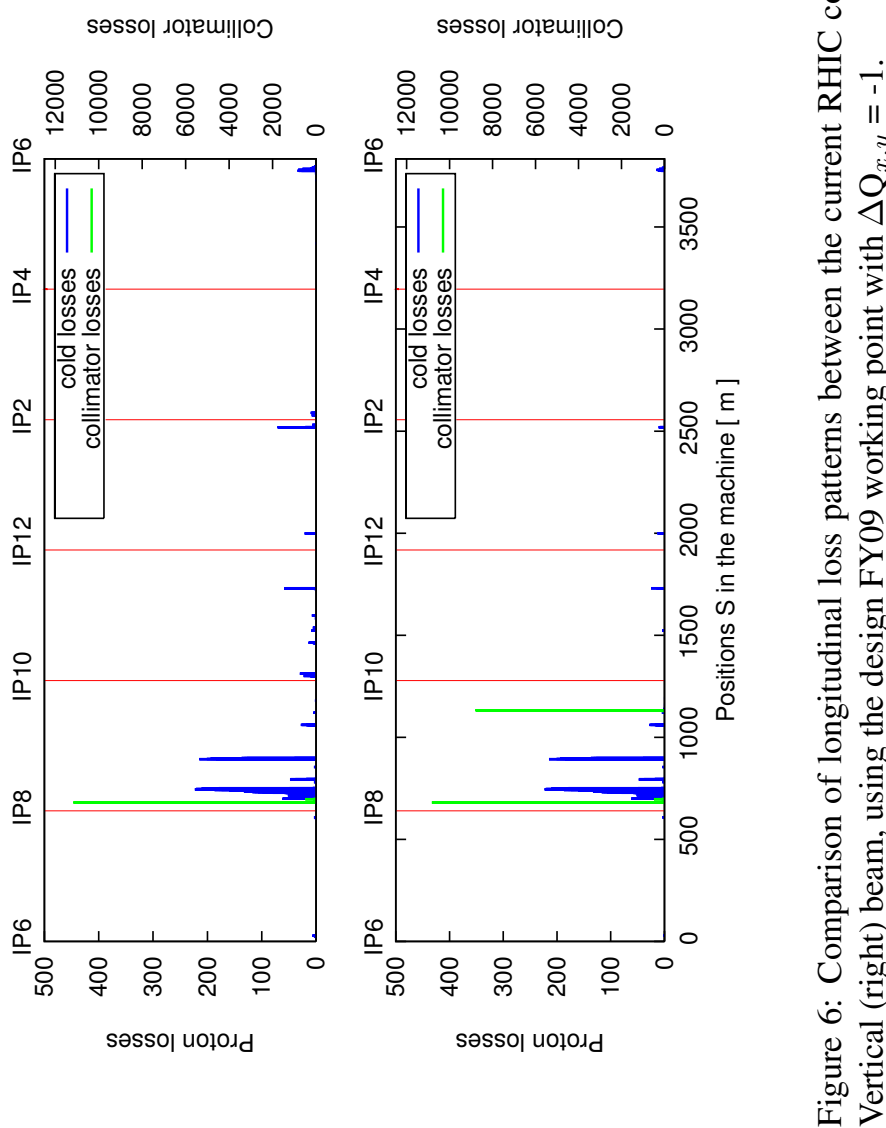

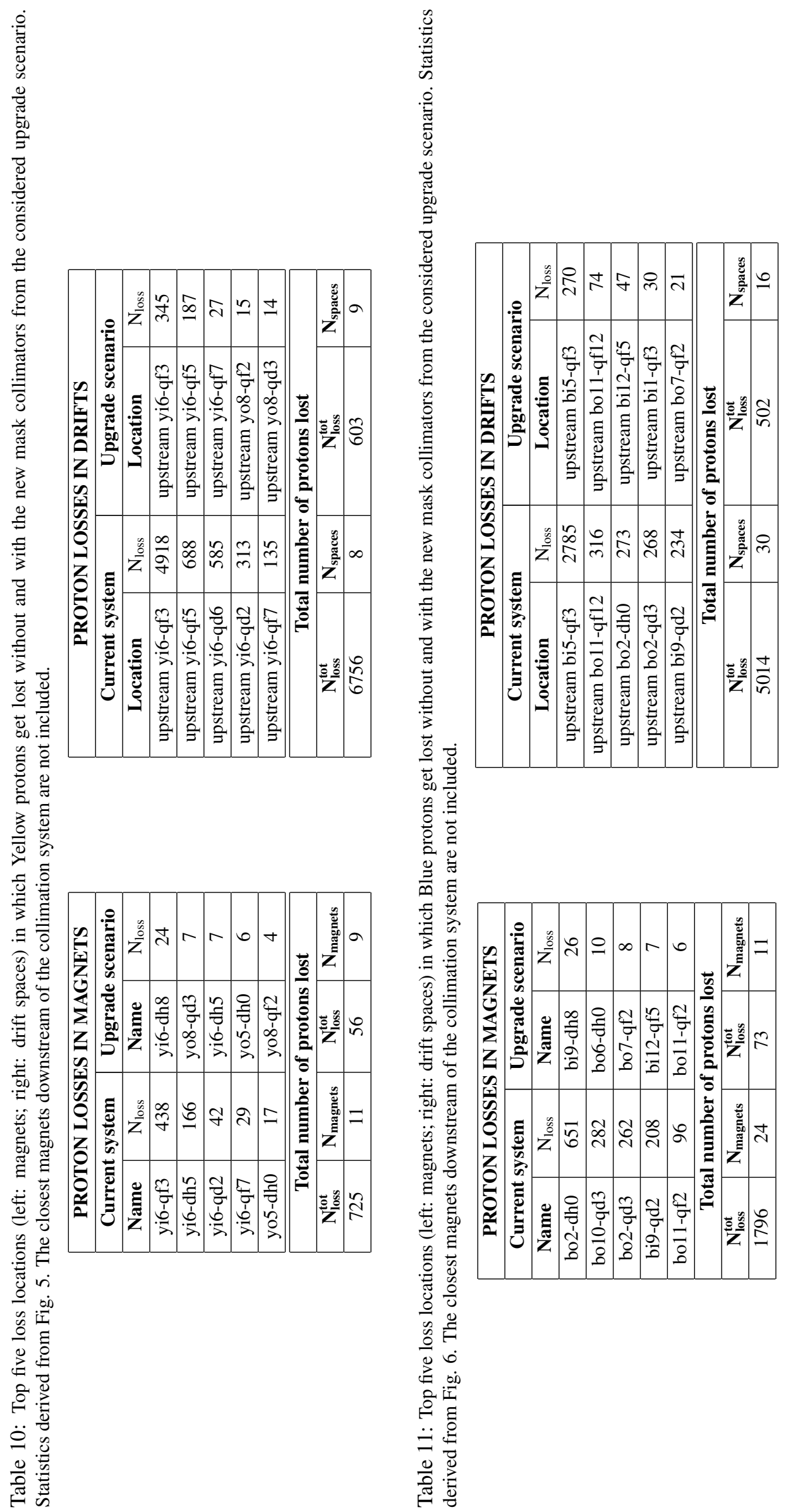